\title{
Lgr5 and stem/progenitor gene expression in gastric/gastroesophageal junction carcinoma - significance of potentially retained stemness
}

\author{
Ju-Yoon Yoon ${ }^{1}$, Christine Brezden-Masley ${ }^{2}$ and Catherine J. Streutker ${ }^{1,3^{*}}$
}

\begin{abstract}
Background: Gastric/gastroesophageal junction (GEJ) adenocarcinomas are heterogeneous, comprising four molecularly distinct subtypes, namely EBV-positive, microsatellite instability (MSI), chromosomal instability (CIN) and genomically stable (GS) subtypes, and a part of this heterogeneity may hypothesized to be different cells-of-origin. Stem/progenitor cell hierarchy in the stomach is complex, which include the Lgr $^{(+)}$gastric stem cells (GSCs).

Methods: While previous studies have focused on non-nuclear Lgr5 expression, nuclear Lgr5 expression has been reported in a subset of stem cells, and we examined nuclear Lgr5 expression in a local cohort of 95 cases of gastric/GEJ adenocarcinoma. mRNA levels for LGR5 and other stem cell marker genes were examined in the TCGA cohort.

Results: We observed nuclear Lgr5 expression in a 18/95 cases. Near mutual exclusivity was seen between nuclear Lgr5 and strong non-nuclear Lgr5. Both strong non-nuclear and nuclear Lgr5 expression tended to be seen more frequently with the intestinal histotype and approximated CIN molecular subtype. With respect to overall survival (OS), nuclear Lgr5 expression appears to be protective, with the worst survival being seen in the cases lacking nuclear Lgr5 and with low non-nuclear Lgr5 expression. When compared to other stem/ progenitor cell markers, LGR5 mRNA expression clusters with other GSC marker genes, including VIL1. Higher expression of these GSC marker genes was associated with better OS.

Conclusions: Our results show that Lgr5 expression is dynamic in gastric/GEJ adenocarcinoma and heterogeneous across the several disease attributes. We postulate that this may reflect "retained stemness" in the form of Lgr $5^{\text {High }}{ }_{\text {GSC }}$ signature that appears to be associated with better survival.
\end{abstract}

Keywords: Lgr5, Gastric/gastroesophageal junction carcinoma, Stem/progenitor cell

\footnotetext{
* Correspondence: cathy.streutker@unityhealth.to

${ }^{1}$ Department of Laboratory Medicine and Pathobiology, University of

Toronto, Toronto, Ontario, Canada

${ }^{3}$ Department of Pathology, St. Michael's Hospital, St. Michael's Hospital, Unity

Health Toronto, Rm 2-099CC, 30 Bond Street, Toronto, Ontario M5B-1W8,

Canada

Full list of author information is available at the end of the article
}

(c) The Author(s). 2020 Open Access This article is licensed under a Creative Commons Attribution 4.0 International License, which permits use, sharing, adaptation, distribution and reproduction in any medium or format, as long as you give appropriate credit to the original author(s) and the source, provide a link to the Creative Commons licence, and indicate if changes were made. The images or other third party material in this article are included in the article's Creative Commons licence, unless indicated otherwise in a credit line to the material. If material is not included in the article's Creative Commons licence and your intended use is not permitted by statutory regulation or exceeds the permitted use, you will need to obtain permission directly from the copyright holder. To view a copy of this licence, visit http://creativecommons.org/licenses/by/4.0/. The Creative Commons Public Domain Dedication waiver (http://creativecommons.org/publicdomain/zero/1.0/) applies to the data made available in this article, unless otherwise stated in a credit line to the data. 


\section{Synopsis}

Significance of Lgr5 expression in gastric/GE junction carcinomas is re-examined in the context of their molecular heterogeneity. Both nuclear and non-nuclear Lgr5 tended to be associated with the intestinal histotype, chromosome instability molecular subtype. LGR5 mRNA expression cluster was associated with better prognosis. High Lgr5/LGR5 expression is speculated to be a retained phenotype that may be reflecting the cellular origin in a subset of gastric carcinomas.

\section{Background}

Gastric/gastro-esophageal junction (GEJ) adenocarcinomas comprise a morphologically, molecularly and clinically diverse group of cancers. TCGA studies demonstrated that gastric/GEJ carcinomas are a heterogeneous group of diseases that could be sub-categorized into four molecular subtypes, namely EBV-related, microsatellite instability (MSI), chromosomal instability (CIN) and genomically stable (GS) [1, 2]. While the molecular heterogeneity to some extent reflects the different etiologies and pathways of tumorigenesis, such as deficiency in the mismatch repair process (leading to the MSI subtype tumors), another potential factor contributing to the molecular diversity in gastric/GEJ adenocarcinoma is the poorly explored concept of "cell-of-origin".

Applying the cell-of-origin concept to gastric/GEJ adenocarcinoma pathogenesis is challenging, related as it is to the complex biology of stem/progenitor cells in the stomach. The gastric mucosa is a site of dynamic regenerative homeostasis, with turnover that is thought to be maintained by long-lived stem/precursor cells, with a number of studies that point to $\operatorname{Lgr} 5^{(+)}$cells as the selfrenewing, multipotent stem cells $[3,4]$. The key signaling pathway behind this phenomenon is thought to be the canonical Wnt pathway, with Lgr5 functioning to potentiate Wnt/ $\beta$-catenin signalling by interacting with R-spondin, among other factors $[5,6]$. This role of Lgr5 is presumably facilitated by its trans-membrane domain allowing for membranous localization, but Lgr5 is a spatially dynamic protein, with expression seen also in the cytoplasm and even the nucleus [7-9]. Surface Lgr5 expression is partially regulated by endocytosis, and inhibiting clathrinmediated endocytosis of Lgr5 diminishes intestinal epithelial cell fitness in murine models [8]. In the correct in vitro conditions, isolated single $\operatorname{Lgr} 5^{(+)}$stem cells have the remarkable capacity to form organoids, known as "mini-guts" [3]. Long-term ablation of Lgr $5^{(+)}$cells impairs epithelial homeostasis in the corpus [10]. In homeostasis, the majority of the $\operatorname{Lgr} 5^{(+)}$stem cells appear to divide symmetrically [11], and their proliferation can be accelerated by $H$. pylori infection [12]; this may represent a potential link between the epidemiological observation of increased risk of carcinogenesis associated with $H$. pylori infection.
Lgr5 has also been postulated to be a marker for cancer stem cells (CSCs) in gastric cancer [10, 13], although the hypothesis is currently lacking in definitive experimental results. While concepts may be overlapping, CSCs differ from cells-of-origin in that CSCs generally refer to progenitor/stem-like cells isolated from cancer specimens, generally with ability to form tumors in xenograft models [14]. CSCs that have been obtained and characterized from the stomach have been isolated using a number of different mesenchymal stem cell (MSC) markers such as CD90 [15-17]. Interestingly, more primitive MSC/MSClike cells are also found in normal stomach, and some studies have attributed roles in intestinal and gastric homeostasis to these MSC/MSC-like cells [16].

When analyzed agnostic to the intrinsic heterogeneity in gastric/GEJ adenocarcinoma, Lgr5 expression does not carry a statistically significant prognostic weight, although some prognostic significance is seen in early stage (I/II) patients [18, 19]. Studies have associated higher Lgr5 expression with more frequent nuclear $\beta$ catenin expression, a marker of active Wnt signalling, and higher Lgr5 has been associated with worse survival in a subset of patients. However, these studies predated the TCGA molecular subtyping, and cellular sublocalization of Lgr5 is now better characterized; nuclear Lgr5 has been reported in hair follicle stem cells, and their expression was observed to be limited to cycling hair follicle stem cells, after asymmetric self-renewal division [7]. In light of these previous reports and better understanding of Lgr5 biology, we examined the prognostic value of LGR5 mRNA and Lgr5 protein, including its nuclear expression, and we frame the discussion to focus on the potential implication in histogenesis.

\section{Methods}

\section{Tissue microarray and immunohistochemistry}

This study was performed in conjunction with our institution's research ethics board (REB 10-280). We studied a cohort that has been described in our previous reports $[20,21]$, where we identified cases of gastric/GEJ carcinoma patients treated at the St. Michael's Hospital (SMH, Toronto, Ontario, Canada) with either gastrectomy or endoscopic mucosal resection, between the period 2001 to 2011. A tissue microarray (TMA) was constructed as described previously [20], consisting of two $0.6 \mathrm{~mm}$ cores per each tumour, with several corresponding normal cores. Histology subtypes were obtained from the pathology reports associated with each case, and diffuse histology was interpreted as per the Lauren classification. Any cases with mixed histology were categorized as "other".

Lgr5 immunohistochemistry was performed using a rabbit monoclonal antibody, clone EPR3065Y (Abcam, catalogue number Ab75850), with 1/100 dilution, 
incubated overnight. Binding of the primary antibody was subsequently detected using ImmPRESS Anti-Rabbit IgG (Vector, catalogue number MP - 7401) and the diaminobenzidine substrate kit (DAKO, catalogue number K3468). Non-nuclear Lgr5 staining intensity and frequency was scored by applying the Allred method [22]. Briefly, the method combines staining intensity (0-3) with staining frequency $(0-5$, with 5 representing $>66 \%$ of cells staining), for maximum score of 8 . The method was modified to examine cytoplasmic/membranous (vs. nuclear) staining, and the scores were converted to "low" (Allred score $\leq 6)$, and "high" (>6).

\section{Approximation of the molecular subtypes}

We had previously described our method for approximation of the molecular subtype [21]. Briefly, we employed a subtyping algorithm based on the TCGA algorithm, a series of dichotomizing steps. We first identified the EBVCIMP cases, identified by EBER in-situ hybridization positivity. The MSI subtypes were next identified through immunohistochemistry (IHC) for mismatch repair (MMR) pathway proteins, MLH1, PMS2, MSH2 and MSH6. Among the remaining MMR intact, EBER-negative cases, GS and CIN subtypes were distinguished based on the histotypes (diffuse vs. Intestinal/mixed, respectively).

\section{Identification of and analysis of stem/progenitor cell marker genes}

A list of nineteen genes were generated through literature review, starting with review papers on gastric stem cells and cancer stem cells in gastric cancer [15, 23]. Some markers were excluded due to lack of interpretable values from the TCGA mRNA levels (z-score values, RNA Seq V2) (ex. CD24, BMI1). SSEA-3 and SSEA-4 are non-protein markers and were substituted with ST3GAL2, which encodes the enzyme responsible for conversion of SSEA-3 to SSEA-4 [24]. The TCGA data were downloaded from cBioPortal (http://www.cbioportal.org/) in the form of mRNA z-score values (RNA Seq V2 RSEM), restricting to the cases that were part of the 2014 TCGA publication [2]. All clinical and pathological data for the TCGA cohort were downloaded from cBioPortal.

\section{Clustering and statistics}

Unsupervised clustering of mRNA expression levels/patterns were performed using Cluster 3.0, and visualized using TreeView [25]. The obtained hierarchical clustering results were used in grouping the TCGA cohort into groups A-C. Survival analysis was performed using the Kaplan-Meier method. Comparisons of continuous variables between multiple groups were performed using analysis of variance (with Tukey post hoc tests). Comparisons of categorical variables between multiple groups were performed using Chi-square test. All statistical tests were performed using JMP (SAS version 13/14).

\section{Results \\ Lgr5 expression patterns in gastric/GEJ carcinoma}

We examined Lgr5 protein expression in our previously described Toronto (St. Michael's Hospital) TMA cohort of gastric adenocarcinoma cases [20, 21]. Non-nuclear (cytoplasmic/membranous) staining intensity was variable, ranging from being completely barely perceptible, low staining (low) to diffuse, strong positivity (high) (Fig. 1a, left). Careful examination of the cores showed that nuclear Lgr5 expression can be seen in 18/95 cases of gastric/GEJ adenocarcinoma (Fig. 1a, right). Areas could be identified showing clear overlap between nuclear Lgr5 expression and frank nuclear atypia, indicating nuclear expression by carcinoma cells (vs. entrapped non-tumour cells). Nuclear staining distribution was relatively bimodal, being either none/very focal (less than $1 \%$ of lesional cells, i.e. negative) or patchy, weak-to-moderate expression in more than $1 \%$ of lesional cells (i.e. positive). Cases with high non-nuclear staining generally lacked nuclear Lgr5 staining ( $p=$ 0.0043) (Fig. 1b). Interestingly, one case with high nonnuclear Lgr5 and nuclear Lgr5 comprised a TMA core containing both better differentiated (gland-forming) areas with high non-nuclear Lgr5 and another area with nuclear Lgr5 seen in the more solid, higher grade area (Supp. Figure 1).

In gastric adenocarcinoma, pathologists assess "differentiation", based on the lesion's resemblance to normal stomach histology with respect to the proportion of the tumor forming well-organized glandular structures, ranging from 1 (well-differentiated) to 4 (undifferentiated). When Lgr5 expression was examined across the differentiation spectrum, high cytoplasmic/membranous staining was seen more frequently with well-to-moderately differentiated (i.e. grade 1,2 ), compared to poorly to undifferentiated (i.e. grade 3, 4) $(p=0.0112$, Fig. 1c), and these results are in line with the previously reported observation [18]. Similarly, nuclear Lgr5 positivity was seen more frequently with better differentiated cancers (8/34 in grade 1-2 vs. 10/61 in grade 3-4), but the difference was not statistically significant (Fig. 1d). A related concept is histotyping of gastric adenocarcinoma; by Lauren classification, gastric adenocarcinoma is divided into intestinal, diffuse and mixed types [26]. In the TCGA cohort, the largest range for LGR5 mRNA levels was observed in the intestinal group, but differences were not statistically significant (Supp. Figure 2). In the SMH cohort, Lgr5 protein expression (both nuclear and non-nuclear) was seen most frequently in the intestinal histotype, and the difference approached statistical significance $(p=0.0507)$.

We next examined Lgr5 protein/LGR5 mRNA across the different molecular subtypes. In the TCGA cohort, 


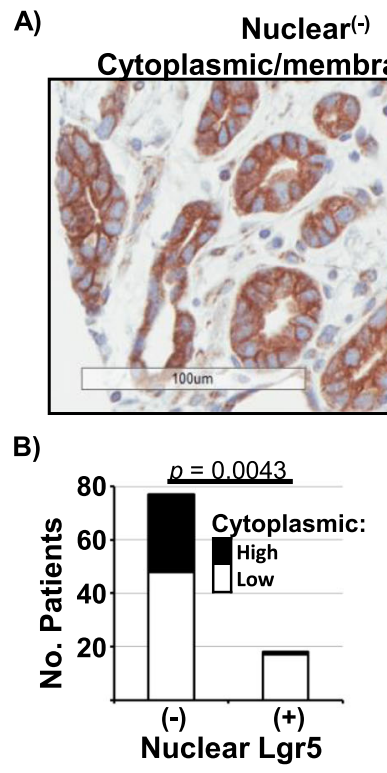

E)

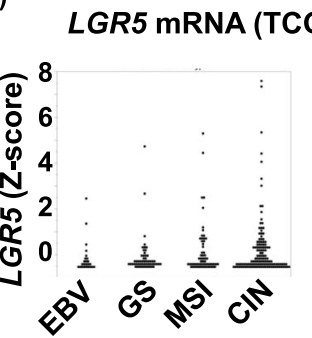

C)

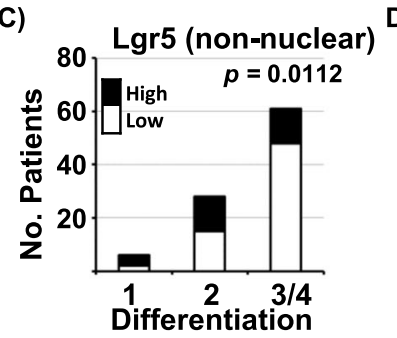

F)

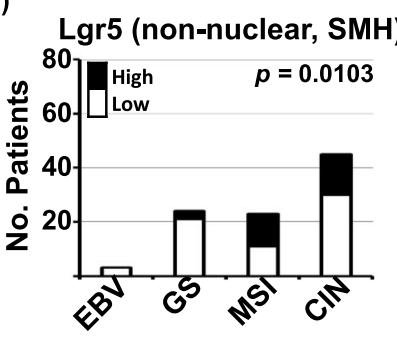

Nuclear ${ }^{(+)}$

Cytoplasmic/membranous Low

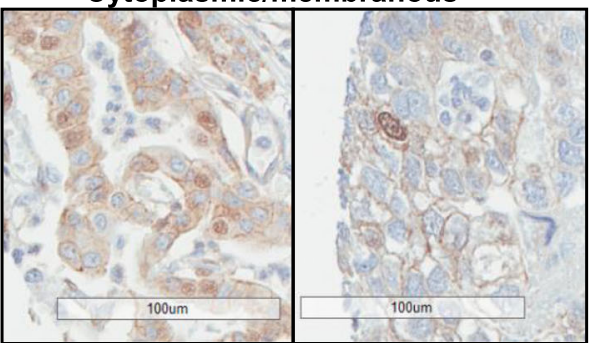

D)

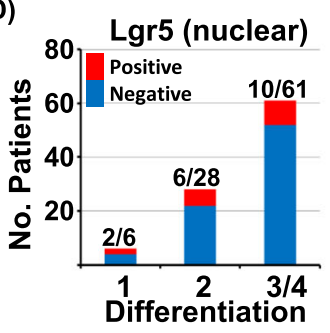

G)

Lgr5 (nuclear, SMH)

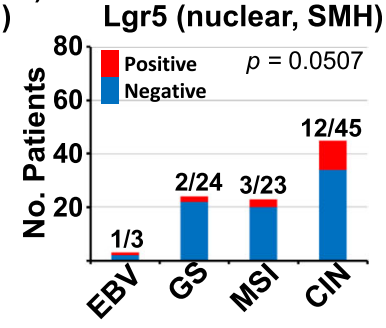

Fig. 1 a Representative $I H C$ sections, showing nuclear and cytoplasmic/membranous Lgr5 staining. Bar $=100 \mu$. b Relationship between nuclear and non-nuclear (cytoplasmic and membranous) Lgr5 levels in the SMH TMA cohort. c Non-nuclear Lgr5 expression with respect to tumor differentiation scores. d Nuclear Lgr5 intensity with respect to differentiation. e Comparison of LGR5 mRNA levels amongst the indicated TCGA molecular subtypes in the TCGA cohort. $\mathbf{f}$ and $\mathbf{g}$ Comparison of non-nuclear $(\mathbf{f})$ and nuclear $(\mathbf{g})$ Lgr5 protein expression levels amongst the approximate molecular subtypes in the SMH cohort. Statistically significant $p$ values are indicated

EBV subtype expressed the lowest levels of LGR5 mRNA, with one comparison (CIN vs. EBV subtypes) being statistical significant $(p=0.0451)$ (Fig. 1e). Examining the protein levels in the SMH cohort by approximated molecular subtypes, cases with strong cytoplasmic Lgr5 were most frequently seen with the CIN and MSI subtypes $(p=0.0103)$ (Fig. 1f). Most of the cases with nuclear Lgr5 expression (12/18 cases with positive nuclear expression) were of the CIN subtype (Fig. 1g), but this difference did not reach statistical significance.

\section{Stem/progenitor cell marker mRNA expression patterns and different subtypes of gastric/GEJ carcinoma}

The stomach is home to several distinct stem/progenitor cell populations, with Lgr5 marking just one of the subpopulations. A number of markers have been used to sort and trace these gastric stem cells (GSCs), as well as cancer stem cells (CSCs) from gastric cancer samples $[15,23]$. Nineteen stem/progenitor cell marker genes were selected based on literature review and TCGA expression data availability (see methods). Examining the mRNA levels in the TCGA cohort, correlation clustering showed two groups, 1) LGR5-containing cluster (cluster $\# 1$ ), which included a number of other well-established gastric stem cell (GSC) markers, such as SOX2 and VIL1, and 2) THY1 (CD90)-containing cluster (cluster \#2), which contained a number of genes that have been established as either mesenchymal stem cell (MSC) markers and/or CSC markers, including CD44, PDGFRB (encoding CD140b), as well as more recently characterized GSC markers, RSPO1 and RSPO3 (Fig. 2a). The strongest positive correlations with LGR5 mRNA levels were seen with TNFRSF19 (encoding TROY, Pearson correlation coefficient $(\mathrm{PCC})=0.2517, p<0.0001)$ and VIL1 (encoding Villin, $\mathrm{PCC}=0.2185, p=0.0003$ ). The strongest positive correlations with THY1 were with PDGFRB $(\mathrm{PCC}=0.7482, p<0.0001)$ and RSPO3 $(\mathrm{PCC}=$ $0.425, p<0.0001)$. 


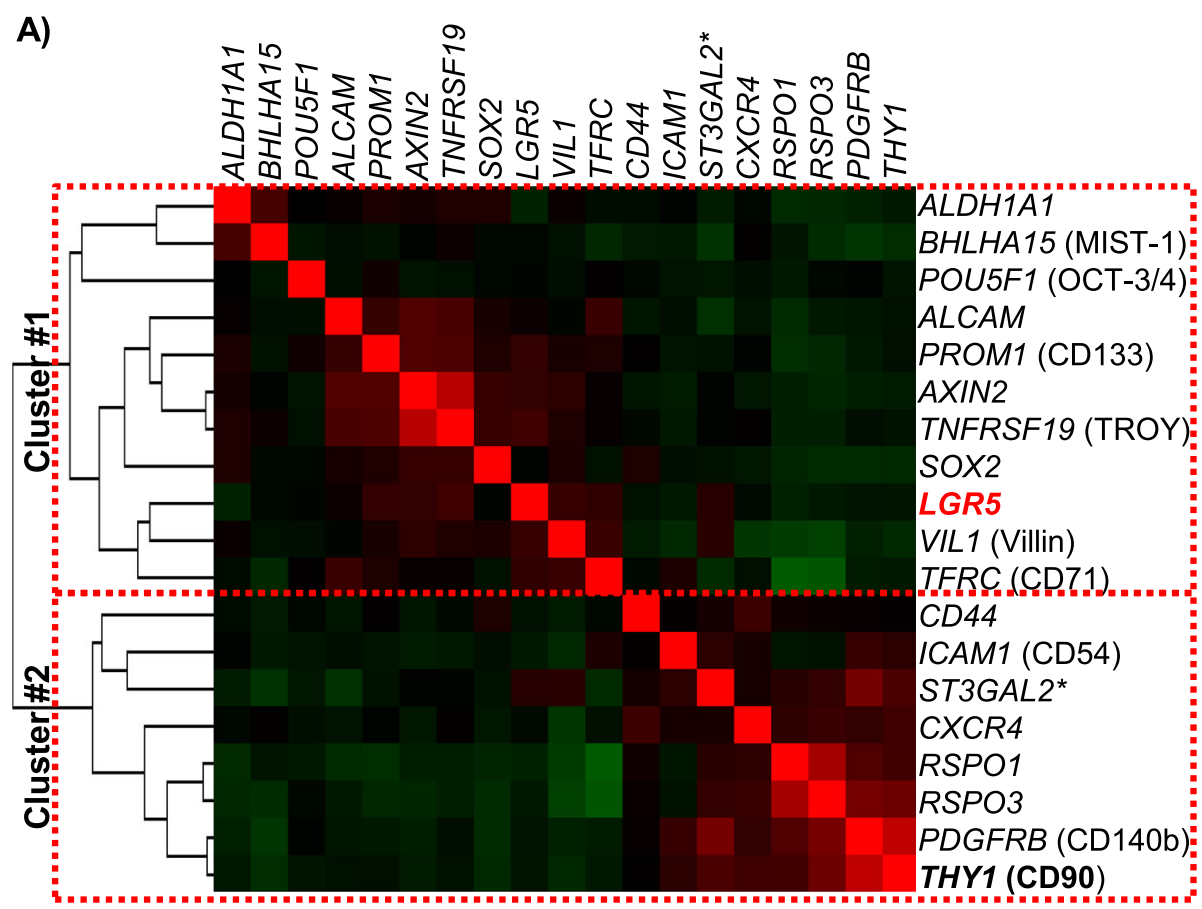

$-1.0$

Correlation

B)

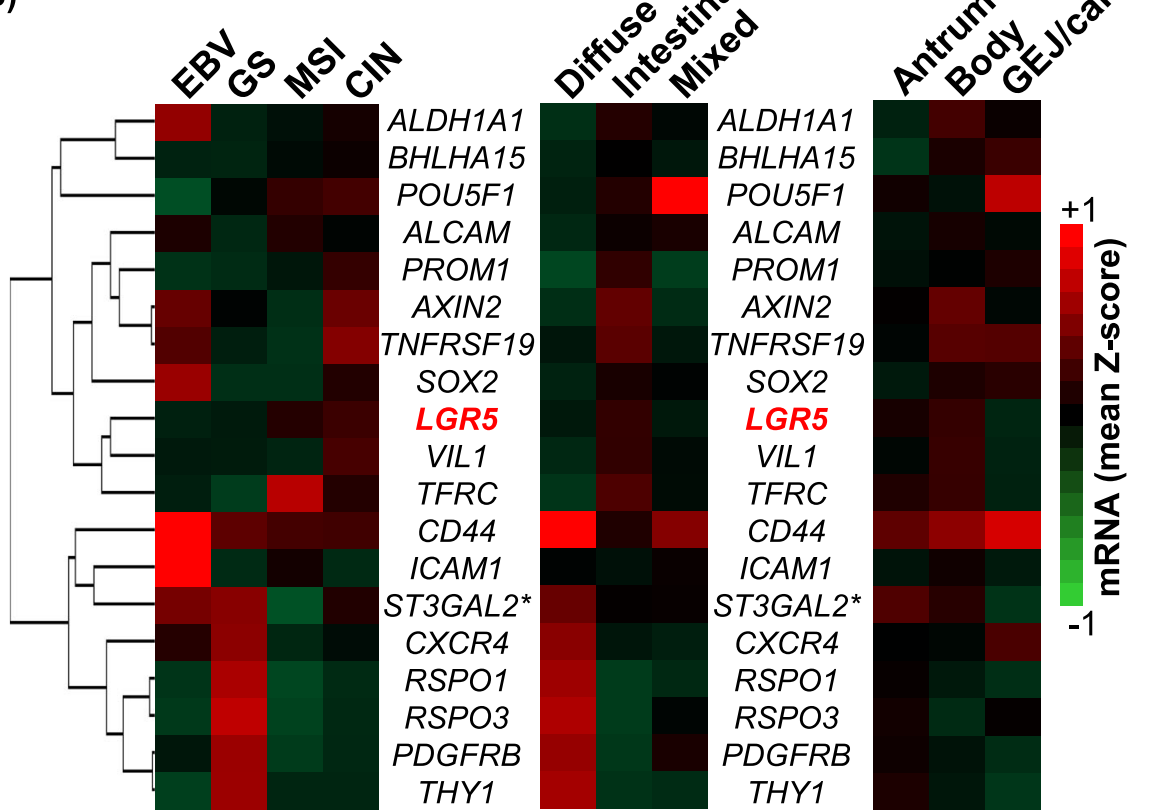

Fig. 2 Stem/progenitor cell marker expression pattern and different subtypes of gastric/GEJ carcinoma. a Clustered correlation heatmap, displaying the correlation coefficients between the nineteen different stem/progenitor cell markers. $\mathbf{b}$ Mean expression levels (Z-score values) for the nineteen stem/progenitor cell markers in the different molecular subtypes (left), histotypes (center) and anatomical tumor epicenter (right)

We next examined the mRNA levels of the same nineteen genes across different molecular subtypes and histological subtypes in the TCGA cohort (Fig. 2b). As expected from the mRNA clustering patterns, the subtypes with higher expression levels of cluster
\#1 genes (containing LGR5) tended to express lower levels of cluster \#2 genes (containing THY1). In particular, the CIN molecular subtype and intestinal histotypes were notable for highest expression levels of the cluster \#1 genes. Cluster \#2 genes were expressed 
at higher levels in the GS molecular subtype and diffuse histotypes.

The stomach is divided into different anatomical regions, namely the GEJ, body/fundus and the antrum/pylorus, and the incidence of different molecular subtypes differ based on the anatomic location within the stomach (Supp. Figure 3A and [1, 2]). When we compared the TCGA cohort by their anatomical origin, the cluster \#1 genes tended to be expressed at higher levels in the fundus/body tumors compared to those from other regions, while higher cluster \#2 gene expression in the antral tumors (Fig. 2b, right). Focusing on LGR5, fundus/ body and antral tumors expressed higher levels of LGR5 mRNA, and the difference was largely driven by CIN tumors (Supp. Figure 3).

\section{Patient survival and stem/progenitor marker expression}

When we examined cytoplasmic/membranous Lgr5 for its prognostic significance, there was no statistically significant difference in overall survival (OS) in our cohort (Supp. Fig. 4), in line with previous studies. By nuclear Lgr5 expression, patients with positive nuclear expression tended to have better OS (Fig. 3a). However, while the number of deaths were markedly different ( $3 / 18$ for nuclear $\operatorname{Lgr} 5^{(+)}$vs. $21 / 77$ for nuclear $\left.\operatorname{Lgr} 5^{(-)}\right)$, the difference was not statistically significant (log-rank $p=$ 0.1742). Noting the relationship between nuclear and cytoplasmic/membranous Lgr5, we next combined the nuclear and cytoplasmic Lgr5 patterns. The worst survival was seen in the cases lacking nuclear Lgr5, with low cytoplasmic/membranous staining (15/48 deaths vs. 9/47 in others), and this difference trended towards statistical significance (log-rank $p=0.0702$ ) (Fig. 3b).

Noting the heterogeneous combination of expression patterns and the two clusters that emerged for stem/progenitor marker genes, we clustered the TCGA cohort based on the expression levels of the nineteen genes. The clustering resulted in 3 groups of cases: A) high expression level of cluster \#1 genes $(n=117), \mathrm{B})$ high expression level of cluster $\# 2$ genes $(n=61)$, and C) low expression of both cluster $\# 1$ and $\# 2(n=87)$ (Fig. 3c). As expected from our results above, group A contained the most number of CIN tumours (69/117), while group B contained the highest number of GS tumours (27/61). Examining patients with known survival data, best OS was seen with group A (median survival 69 months), compared with groups B (median survival 30.9 months) and group C (median survival 24.3 months) (log-rank $p=0.0178$ ) (Fig. 3b). The differences between the survival curves are significant, considering the lack of survival difference by molecular subtypes in the TCGA study [2].

Taken together, gastric/GEJ carcinomas exhibit heterogeneous expression of the different stem/progenitor marker genes. Among them, LGR mRNA and Lgr5 protein expression pattern is dynamic, with respect to cellular localization (nuclear vs. cytoplasmic/membranous) and to tumor pathologic attributes (anatomical epicenter, histotype and molecular subtypes). Higher expression of GSC markers, including LGR5 and VIL1, are seen with $\mathrm{CIN} /$ intestinal subtypes and associated with better survival. This is in contrast to many prior studies that have linked stem cell signatures with worse survival.

\section{Discussion}

The general dogma in many studies that have examined "stemness" posit that stemness is associated with enhanced ability to invade, and thus ultimately being associated with worse survival. However, our data with Lgr5/ LGR5 suggest that this story is likely more complex-it depends on what stem signature is present. The immunohistochemical and mRNA expression profile phenotype with $\operatorname{Lgr} 5^{\text {High }}$-GSC signature combination, which may represent "retained stemness", was associated with better survival (Fig. 3). If this is indeed a phenotype retained from its cell-of-origin, we may speculate that these tumors retained their intrinsic predisposition for glandular differentiation, explaining the association seen with better differentiated, intestinal histo-morphology, and Lgr5 expression. Lgr5 ${ }^{(+)}$cells have been postulated to be the cellular origin of invasive gastric carcinoma by others, specifically in the intestinal histotype [27, 28]. Noting the association with the CIN molecular subtype, the $\operatorname{Lgr} 5^{(+)}$may have an intrinsic predilection for a specific tumorigenic pathway. Targeted deletion of Smad4 and PTEN in $\operatorname{Lgr} 5^{(+)}$gastric stem cells in mice resulted in formation of invasive adenocarcinoma, whereas the same set of genetic maneuvers failed to induce tumorigenesis in differentiated cells, including antral parietal cells, pit cells, as well as corpus $\operatorname{Lgr} 5^{(+)}$chief cells [28]. The likely candidate precursor in this pathway would be intestinal metaplasia (IM), and both $\operatorname{Lgr} 5 \mathrm{mRNA}$ and Lgr5 protein expression has been reported in IM [27, 29, 30]. Lgr5 $5^{(+)}$cells are seen at the crypt base in IM, and the expression becomes more marked in carcinoma in its luminal surface, tumor center and the invasion front type [10, 27]. Interestingly, while spasmolytic polypeptide-expressing metaplasia (SPEM), another putative precursor to gastric adenocarcinoma, was suggested to be not associated with $\operatorname{Lgr} 5^{(+)}$cells in the variegated Lgr5-EGFP-IRES-Cre (ERT2/+); in Rosa26R mice [31], expression of constitutively active Kras (harboring p.G12D hotspot mutation) in Lgr $5^{(+)}$chief cells was sufficient form SPEM in a non-variegated Lgr5-2A-CreERT2 mouse model [10]. Thus, at least two different pathogenic pathways involving Lgr5-expressing cells appear to be possible, both of which may give rise to CIN molecular subtype tumors. Interestingly, while the tumors with GEJ/cardia 


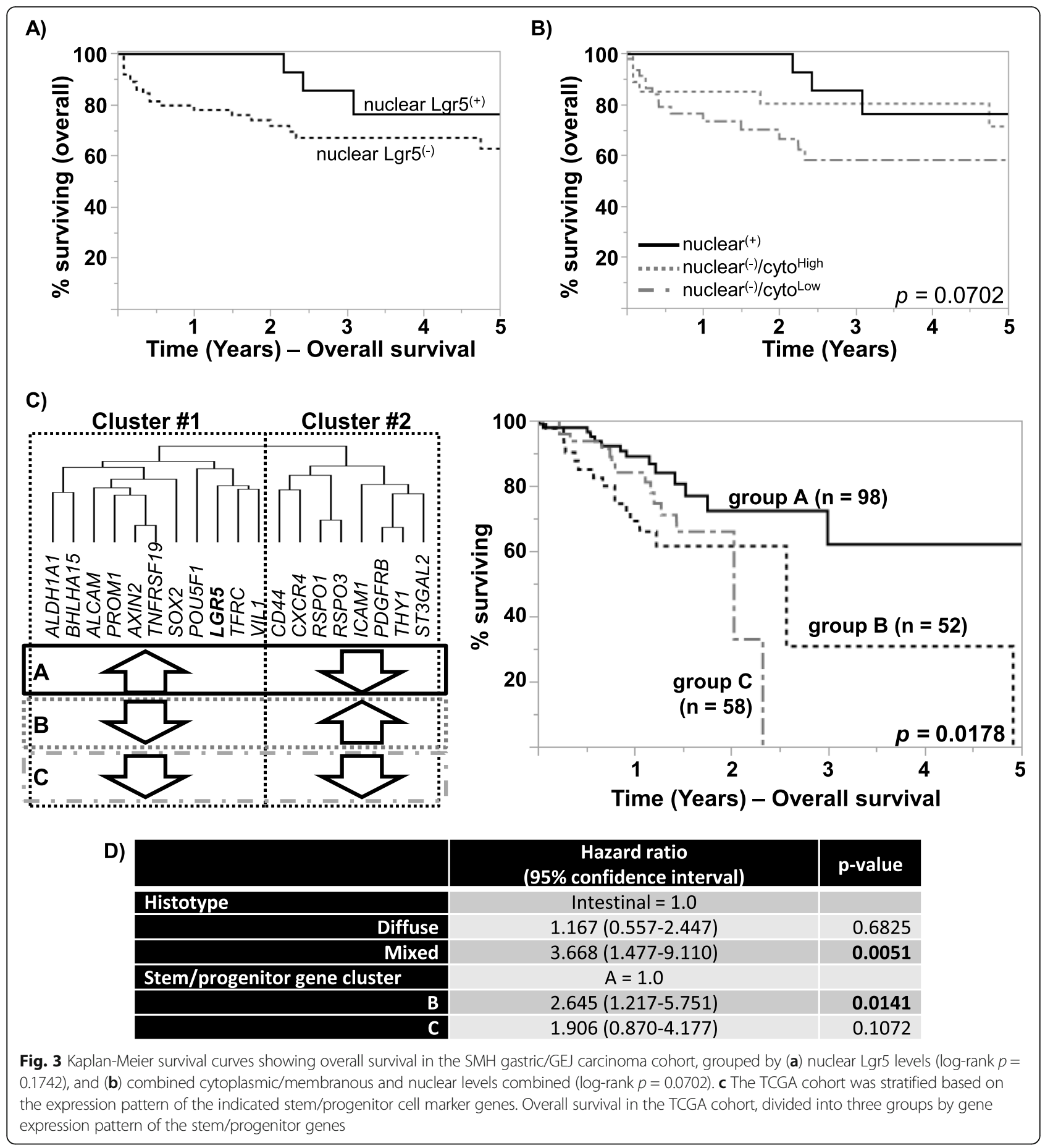

epicenter are largely of the CIN molecular subtype [1], these tumors expressed lower levels of LGR5 mRNA. The abundance of $\operatorname{Lgr} 5^{(+)}$cells varies across the different anatomical regions, being most abundant in the antrum [3, 29]. Thus, there may also be heterogeneity amongst the CIN tumors, with those cardiac/GEJ tumors potentially arising from Lgr5-negative cells, perhaps including the recently described osteopontin responsive, Lgr5-negative/
CD44 ${ }^{(+)}$cells [32]. In line with this possibility, GEJ/cardia tumors were notable for high CD44 mRNA, although CD44 mRNAs are likely to be expressed by a variety of non-tumoral cells, especially lymphocytes.

A part of the challenge in analyzing Lgr5 and its clinical significance is related to its complex biology, including its heterogeneous sub-cellular localization. The physiologic role of nuclear Lgr5 has only thus far been 
described in the hair follicle stem cells, where nuclear Lgr5 expression is limited to the cycling stem cell sister after asymmetric self-renewal divisions [7]. While the role of nuclear Lgr5 in the gastric epithelium and in cancer cells remains largely unexplored, it is worth noting that several studies examining $\operatorname{Lgr} 5^{(+)}$cells based their identification of $\operatorname{Lgr} 5^{(+)}$cells based on LGR5 mRNA expression and/or LGR5-driven reporters (ex. Lgr5-lacZ), where the sub-cellular localization of reporters may differ from that of the endogenous $\operatorname{Lgr} 5[3,10,29]$. Inferring from studies on hair follicle stem cells [7], nuclear Lgr5 may be highlighting the cancer cells with a yet a different pattern of "stemness". While nuclear Lgr5 was nearly mutually exclusive with non-nuclear, strong Lgr5 expression, nuclear Lgr5 also showed similar associations with the intestinal histotype and CIN molecular subtype, suggesting nuclear Lgr5 may also represent a pattern of "retained" phenotype, similar to nonnuclear Lgr5.

Beside the Lgr $5^{(+)} / L G R 5$-expressing cells, more primitive MSC/MSC-like cells appear to be playing a crucial role in intestinal homeostasis, and MSC-like cells have been isolated from human gastric cancer tissues, marked by high expression of THY1 [16, 17]. Some studies have even implicated bone marrow-derived MSCs in gastric epithelium homeostasis, which have been shown to repopulate the gastric epithelium [33, 34]. Interestingly, bone marrow-derived MSCs, at least in mouse models, appear to be able to give rise to both the carcinoma cells and the cancer-associated stromal cells, contributing to disease progression $[33,34]$. In the context of these theories of gastric cancer carcinogenesis, the clustering pattern of different stem/progenitor cell markers seen in the TCGA data is interesting (Fig. 2a), which suggest that there may be at least two different signatures of stem/progenitor gene expression, i.e. one more intestinal (cluster \#1, LGR5-high) and one more MSC-like (cluster \#2, THY1-high). However, this is likely to be a simplified version of the story, considering the complex hierarchy between the different stem/progenitor cells in the GI tract $[35,36]$. Future studies may thus benefit from examining co-expression patterns of multiple markers, including Bmil, which appear to mark slow-cycling stem/progenitor cells that can revert to express Lgr5 [35, $37,38]$, while noting the different sub-cellular Lgr5 expression patterns. One peculiar finding was the coclustering of RSPO1 and RSPO3 in cluster \#2. The mRNA levels of the two genes correlated highly with one another, while correlating little or negatively with the cluster \#1 genes (Fig. 2a). R-spondins are ligands to Lgr5, and the "Rspo-Lgr5 axis" plays an important role in stimualting the growth of intestinal crypts in vitro via the Wnt pathway [39, 40]. While the "Rspo-Lgr5 axis" had been previously postulated to be merely an amplification process for Wnt signaling, more recent studies point to non-overlapping, non-interchangeable roles between Wnt and R-spondin in GSCs [41]. As well, R-spondins, at least R-spondin 3, can be produced by myofibroblasts in the stomach [6]. As fibroblasts/myofibroblasts are also marked by CD90 (Thy1) expression, these observations may be merely indicating enrichment of stromal cells. Alternatively, Lgr5, perhaps in its nuclear form, may be carrying out functions independent of R-spondins in gastric/GEJ carcinoma, and further studies would be required to better understand the Rspo-Lgr5 axis in gastric/GEJ carcinoma.

We encountered several challenges in this study, one of which was related to our relatively small cohort size, which likely explains the lack of statistical significance with some of the analyses (ex. OS analysis in Fig. 3a/b). Another weakness of this study is its purely correlative nature, and we cannot differentiate between retained vs. acquired Lgr5 expression. Molecular profiling of $\operatorname{Lgr} 5^{(+)} /$ LGR5-expressing cell-derived tumors through the pathogenic pathway, including the precursors, could shed further light on the tumorigenic pathway. $\operatorname{Lgr} 5^{(+)} / L G R 5$ expressing cell-derived tumors may not be restricted to a specific molecular subtype, and CIN-type tumors are unlikely to be restricted to $\operatorname{Lgr} 5^{(+)} / L G R 5$-expressing cells as their sole cell-of-origin. The hierarchy of the stem/ progenitor cells in the stomach await further clarification, and we await identification of other candidate cellsof-origin and to assess the impact of genetic manipulation of other candidate cells.

\section{Conclusions}

Our results show that Lgr5 expression is dynamic in gastric/GEJ adenocarcinoma and heterogeneous across the several disease attributes. We postulate that this may reflect "retained stemness" in the form of $\operatorname{Lgr} 5^{\mathrm{High}}-\mathrm{GSC}$ signature that appears to be associated with better survival.

\section{Supplementary information}

Supplementary information accompanies this paper at https://doi.org/10. 1186/s12885-020-07362-7.

\section{Additional file 1 \\ Additional file 2. \\ Additional file 3 . \\ Additional file 4}

\section{Abbreviations}

CIN: Chromosomal instability; CSC: Cancer stem cell; EBER: EBV-encoded small RNA; EBV: Ebstein-Barr Virus; GEJ: Gastroesophageal junction; GS: Genomically stable; IHC: Immunohistochemistry; GSC: Gastric stem cell; MSC: Mesenchymal stem cell; MSI: Microsatellite instability; MMR: Mismatch repair; OS: Overall survival; SMH: St. Michael's Hospital; TCGA: The cancer genome atlas; TMA: Tissue microarray 


\section{Acknowledgements}

The authors would like to acknowledge the assistance of Ms. Vidya Beharry who assisted with immunohistochemistry testing.

\section{Authors' contributions}

JYY co-designed the project and performed the majority of the acquisition and interpretation of the data and was the primary manuscript writer. CBM contributed to acquisition of data and revised the manuscript. CJS codesigned the project with JYY, worked on acquisition and interpretation of data and revised the manuscript. All authors have read and approved the manuscript.

\section{Funding}

The study was funded by an unrestricted grant from the Laboratory Associates Group of St. Michael's Hospital, Unity Health Toronto; the funding group had no other role in the project.

\section{Availability of data and materials}

The datasets used and/or analysed during this study are available from the corresponding author on reasonable request.

\section{Ethics approval and consent to participate}

This study was performed with approval from the St. Michael's Hospital/Unity Health Network Research Ethics Board (REB 10-280). The need for informed consent was waived by the SMH/Unity Health Toronto Research Ethics Board.

\section{Consent for publication}

Not Applicable.

\section{Competing interests}

The authors declare that they have no competing interests.

\section{Author details}

'Department of Laboratory Medicine and Pathobiology, University of Toronto, Toronto, Ontario, Canada. ${ }^{2}$ Department of Hematology/Oncology, Mount Sinai Hospital, Toronto, ON, Canada. ${ }^{3}$ Department of Pathology, St. Michael's Hospital, St. Michael's Hospital, Unity Health Toronto, Rm 2-099CC, 30 Bond Street, Toronto, Ontario M5B-1W8, Canada.

\section{Received: 10 March 2020 Accepted: 31 August 2020}

\section{Published online: 07 September 2020}

\section{References}

1. The Cancer Genome Atlas Research N. Integrated genomic characterization of oesophageal carcinoma. Nature. 2017;541(7636):169-75.

2. The Cancer Genome Atlas Research N. Comprehensive molecular characterization of gastric adenocarcinoma. Nature. 2014:513(7517):202-9.

3. Barker N, Huch M, Kujala P, van de Wetering M, Snippert HJ, van Es JH, Sato $T$, Stange DE, Begthel $H$, van den Born M, Danenberg E, van den Brink S, Korving J, Abo A, Peters PJ, Wright N, Poulsom R, Clevers H. Lgr5+ve stem cells drive self-renewal in the stomach and build long-lived gastric units in vitro. Cell Stem Cell. 2010;6(1):25-36.

4. Matsuo J, Kimura S, Yamamura A, Koh CP, Hossain MZ, Heng DL, Kohu K, Voon DC-C, Hiai H, Unno M, So JBY, Zhu F, Srivastava S, Teh M, Yeoh KG, Osato M, Ito Y. Identification of Stem Cells in the Epithelium of the Stomach Corpus and Antrum of Mice. Gastroenterology. 2017;152(1):218-31.e14.

5. Leung C, Tan SH, Barker N. Recent advances in Lgr $5<$ sup $\rangle+</$ sup $>$ stem cell research. Trends Cell Biol. 2018;28(5):380-91.

6. Sigal M, Logan CY, Kapalczynska M, Mollenkopf HJ, Berger H, Wiedenmann B, Nusse R, Amieva MR, Meyer TF. Stromal R-spondin orchestrates gastric epithelial stem cells and gland homeostasis. Nature. 2017;548(7668):451-5.

7. Huh YH, King J, Cohen J, Sherley JL. SACK-expanded hair follicle stem cells display asymmetric nuclear Lgr5 expression with non-random sister chromatid segregation. Sci Rep. 2011;1:176.

8. Snyder JC, Rochelle LK, Ray C, Pack TF, Bock CB, Lubkov V, Lyerly HK, Waggoner AS, Barak LS, Caron MG. Inhibiting clathrin-mediated endocytosis of the leucine-rich $\mathrm{G}$ protein-coupled receptor-5 diminishes cell fitness. J Biol Chem. 2017;292(17):7208-22.

9. Chen $\mathrm{P}-\mathrm{H}$, Chen $\mathrm{X}$, Lin Z, Fang D, He X. The structural basis of R-spondin recognition by LGR5 and RNF43. Genes Dev. 2013;27(12):1345-50.
10. Leushacke M, Tan SH, Wong A, Swathi Y, Hajamohideen A, Tan LT, Goh J, Wong E, Denil SLIJ, Murakami K, Barker N. Lgr5-expressing chief cells drive epithelial regeneration and cancer in the oxyntic stomach. Nat Cell Biol. 2017;19:774.

11. Leushacke M, Ng A, Galle J, Loeffler M, Barker N. Lgr5+ gastric stem cells divide symmetrically to effect epithelial homeostasis in the pylorus. Cell Rep. 2013;5(2):349-56.

12. Sigal $M$, Rothenberg ME, Logan CY, Lee JY, Honaker RW, Cooper RL, Passarelli B, Camorlinga M, Bouley DM, Alvarez G, Nusse R, Torres J, Amieva MR. $<$ em $>$ Helicobacter pylori $<$ /em $>$ Activates and Expands Lgr $5<$ sup $>+$ $</$ sup $>$ Stem Cells Through Direct Colonization of the Gastric Glands. Gastroenterology. 2015;148(7):1392-404.e21.

13. Wang $B$, Chen $Q$, Cao Y, Ma X, Yin C, Jia Y, Zang A, Fan W. LGR5 is a gastric Cancer stem cell marker associated with Stemness and the EMT signature genes NANOG, NANOGP8, PRRX1, TWIST1, and BMI1. PLoS One. 2016;11(12): e0168904.

14. Rycaj K, Tang DG. Cell-of-Origin of Cancer versus Cancer Stem Cells: Assays and Interpretations. Cancer Res. 75(19):4003-11.

15. Zhao Y, Feng F, Zhou Y-N. Stem cells in gastric cancer. World J Gastroenterol: WJG. 2015;21(1):112.

16. Gong W, Guo M, Han Z, Wang Y, Yang P, Xu C, Wang Q, Du L, Li Q, Zhao H, Fan F, Liu Q. Mesenchymal stem cells stimulate intestinal stem cells to repair radiation-induced intestinal injury. Cell Death Dis. 2016;7(9):e2387.

17. Cao H, Xu W, Qian H, Zhu W, Yan Y, Zhou H, Zhang X, Xu X, Li J, Chen Z, Xu X. Mesenchymal stem cell-like cells derived from human gastric cancer tissues. Cancer Lett. 2009;274(1):61-71.

18. Bu Z, Zheng Z, Zhang L, Li Z, Sun Y, Dong B, Wu A, Wu X, Wang X, Cheng $X$, Xing $X, L i Y$, Du H, Ji J. LGR5 is a promising biomarker for patients with stage I and II gastric cancer. Chin J Cancer Res. 2013;25(1):79-89.

19. Jang $B G$, Lee $B L$, Kim WH. Prognostic significance of leucine-rich-repeatcontaining G-protein-coupled receptor 5, an intestinal stem cell marker, in gastric carcinomas. Gastric Cancer. 2016;19(3):767-77.

20. Wiegand KC, Sy K, Kalloger SE, Li-Chang H, Woods R, Kumar A, Streutker CJ, Hafezi-Bakhtiari S, Zhou C, Lim HJ, Huntsman DG, Clarke B, Schaeffer DF. ARID1A/BAF250a as a prognostic marker for gastric carcinoma: a study of 2 cohorts. Hum Pathol. 2014;45(6):1258-68.

21. Yoon J-Y, Streutker C. Five-stain-based approximation of molecular subtyping in gastric adenocarcinoma and its prognostic significance in a single institution cohort. Manuscript in preparation.

22. Harvey JM, Clark GM, Osborne CK, Allred DC. Estrogen receptor status by immunohistochemistry is superior to the ligand-binding assay for predicting response to adjuvant endocrine therapy in breast cancer. J Clin Oncol. 1999; 17(5):1474.

23. Kim T-H, Shivdasani RA. Stomach development, stem cells and disease. Development. 2016;143(4):554-65.

24. Saito S, Aoki H, Ito A, Ueno S, Wada T, Mitsuzuka K, Satoh M, Arai Y, Miyagi T. Human alpha2,3-sialyltransferase (ST3Gal II) is a stage-specific embryonic antigen-4 synthase. J Biol Chem. 2003;278(29):26474-9.

25. de Hoon MJ, Imoto S, Nolan J, Miyano S. Open source clustering software. Bioinformatics. 2004;20(9):1453-4.

26. Bosman FT. Carneiro F. Theise ND. WHO Classification of Tumours of the Digestive System. Fourth Edition: Hruban RH; 2010.

27. Simon E, Petke D, Böger C, Behrens H-M, Warneke V, Ebert M, Röcken C. The spatial distribution of LGR5(+) cells correlates with gastric Cancer progression. PLoS One. 2012;7(4):e35486.

28. Li XB, Yang G, Zhu L, Tang YL, Zhang C, Ju Z, Yang X, Teng Y. Gastric Lgr5(+ ) stem cells are the cellular origin of invasive intestinal-type gastric cancer in mice. Cell Res. 2016;26(7):838-49.

29. Jang BG, Lee BL, Kim WH. Distribution of LGR5+ cells and associated implications during the early stage of gastric tumorigenesis. PLoS One. 2013;8(12):e82390-e.

30. Zheng Z-X, Sun Y, Bu Z-D, Zhang L-H, Li Z-Y, Wu A-W, Wu X-J, Wang X-H, Cheng $X-J$, Xing $X-F, D$ D H, Ji J-F. Intestinal stem cell marker LGR5 expression during gastric carcinogenesis. World J Gastroenterol. 2013;19(46): $8714-21$.

31. Nam KT, O'Neal RL, Coffey RJ, Finke PE, Barker N, Goldenring JR. Spasmolytic polypeptide-expressing metaplasia (SPEM) in the gastric oxyntic mucosa does not arise from Lgr5-expressing cells. Gut. 2012;61(12):1678-85.

32. Fu D-J, Wang L, Chouairi FK, Rose IM, Abetov DA, Miller AD, Yamulla RJ, Schimenti JC, Flesken-Nikitin A, Nikitin AY. Gastric squamous-columnar 
junction contains a large pool of cancer-prone immature osteopontin responsive Lgr5-CD44+ cells. Nat Commun. 2020;11(1):84.

33. Houghton J, Stoicov C, Nomura S, Rogers AB, Carlson J, Li H, Cai X, Fox JG, Goldenring JR, Wang TC. Gastric Cancer originating from bone marrowderived cells. Science. 2004;306(5701):1568-71.

34. Quante M, Tu SP, Tomita H, Gonda T, Wang SSW, Takashi S, Baik GH, Shibata W, DiPrete B, Betz KS, Friedman R, Varro A, Tycko B, Wang TC. Bone marrowderived Myofibroblasts contribute to the Mesenchymal stem cell niche and promote tumor growth. Cancer Cell. 2011;19(2):257-72.

35. Smith NR, Swain JR, Davies PS, Gallagher AC, Parappilly MS, Beach CZ, Streeter PR, Williamson IA, Magness ST, Wong MH. Monoclonal Antibodies Reveal Dynamic Plasticity Between Lgr5- and Bmi1-Expressing Intestinal Cell Populations. Cell Mol Gastroenterol Hepatol. 2018;6(1):79-96.

36. Li N, Yousefi M, Nakauka-Ddamba A, Jain R, Tobias J, Epstein Jonathan A, Jensen Shane T, Lengner CJ. Single-cell analysis of proxy reporter allelemarked epithelial cells establishes intestinal stem cell hierarchy. Stem Cell Reports. 2014;3(5):876-91.

37. Jadhav U, Saxena M, O'Neill NK, Saadatpour A, Yuan G-C, Herbert Z, Murata K, Shivdasani RA. Dynamic Reorganization of Chromatin Accessibility Signatures during Dedifferentiation of Secretory Precursors into Lgr5+ Intestinal Stem Cells. Cell Stem Cell. 2017;21(1):65-77.e5.

38. Yan KS, Gevaert O, GXY Z, Anchang B, Probert CS, Larkin KA, Davies PS, Cheng Z-f, Kaddis JS, Han A, Roelf K, Calderon RI, Cynn E, Hu X, Mandleywala K, Wilhelmy J, Grimes SM, Corney DC, Boutet SC, Terry JM, Belgrader P, Ziraldo SB, Mikkelsen TS, Wang F, von Furstenberg RJ, Smith NR, Chandrakesan P, May R, MAS C, Jain R, Cartwright CA, Niland JC, Hong Y-K, Carrington J, Breault DT, Epstein J, Houchen CW, Lynch JP, Martin MG, Plevritis SK, Curtis C, Ji HP, Li L, Henning SJ, Wong MH, Kuo CJ. Intestinal Enteroendocrine Lineage Cells Possess Homeostatic and Injury-Inducible Stem Cell Activity. Cell Stem Cell. 2017;21 (1):78-90.e6.

39. de Lau W, Barker N, Low TY, Koo BK, Li VS, Teunissen H, Kujala P, Haegebarth A, Peters PJ, van de Wetering M, Stange DE, van Es JE, Guardavaccaro D, Schasfoort RB, Mohri Y, Nishimori K, Mohammed S, Heck AJ, Clevers H. Lgr5 homologues associate with Wnt receptors and mediate R-spondin signalling. Nature. 2011;476(7360):293-7.

40. Carmon KS, Gong X, Lin Q, Thomas A, Liu Q. R-spondins function as ligands of the orphan receptors LGR4 and LGR5 to regulate Wnt/ $\beta$-catenin signaling. Proc Natl Acad Sci. 2011;108(28):11452-7.

41. Yan KS, Janda CY, Chang J, Zheng GXY, Larkin KA, Luca VC, Chia LA, Mah AT, Han A, Terry JM, Ootani A, Roelf K, Lee M, Yuan J, Li X, Bolen CR, Wilhelmy J, Davies PS, Ueno H, von Furstenberg RJ, Belgrader P, Ziraldo SB, Ordonez H, Henning SJ, Wong MH, Snyder MP, Weissman IL, Hsueh AJ, Mikkelsen TS, Garcia KC, Kuo CJ. Non-equivalence of Wnt and R-spondin ligands during Lgr5+ intestinal stem-cell self-renewal. Nature. 2017;545:238.

\section{Publisher's Note}

Springer Nature remains neutral with regard to jurisdictional claims in published maps and institutional affiliations.

\section{Ready to submit your research? Choose BMC and benefit from:}

- fast, convenient online submission

- thorough peer review by experienced researchers in your field

- rapid publication on acceptance

- support for research data, including large and complex data types

- gold Open Access which fosters wider collaboration and increased citations

- maximum visibility for your research: over $100 \mathrm{M}$ website views per year

At BMC, research is always in progress.

Learn more biomedcentral.com/submissions 